\title{
Evaluation of Intracoronary Pressure and Blood Flow Velocity for the Assessment of Coronary Stenoses Severity
}

\author{
C Perrey ${ }^{1}$, W Bojara $^{2}$, M Lindstaedt $^{2}$, C Fischer $^{1,2}$, H Ermert $^{1}$ \\ ${ }^{1}$ Institute of High Frequency Engineering and ${ }^{2}$ Department of Cardiology Bergmannsheil \\ Ruhr University, Bochum, Germany
}

\begin{abstract}
For the assessment of coronary stenoses severity, intracoronary pressure and blood flow velocity were measured simultaneously during catheterization procedures. In 10 patients pressure measurements were performed distal and proximal of the stenoses. Simultaneously, the coronary blood flow velocity was recorded using Doppler ultrasound catheters. Data was recorded at resting conditions and at hyperemia. All signals were digitized at $200 \mathrm{~Hz}$. Mean pressure gradients were plotted versus flow velocity, and linear regression was applied to the data. The slopes of the resulting lines were used as an indicator for stenosis severity.

In addition, a new parameter for the degree of obstruction was derived by frequency analysis of proximal and distal pressure waveforms. For verification, the described methods were compared to clinically established methods.
\end{abstract}

\section{Introduction}

The severity of coronary stenoses is usually assessed invasively by morphological measurements, such as quantitative coronary angiography (QCA) or intravascular ultrasound (IVUS). The recent development of guide wires with miniaturized sensor tips allows the evaluation of additional functional information, namely intracoronary pressure and flow velocity. Various methods have been developed for this task, such as the calculation of myocardial fractional flow reserve (FFR) derived from pressure measurements [1] or coronary flow velocity reserve (CFVR), calculated from Doppler flow measurements [2]. Currently, these functional parameters are only assessed separately in clinical practice and the validity of the measurements depends on a state of maximum flow (maximum hyperemia) [3]. A pressure based parameter that does not require hyperemia was developed by Brosh et. al., evaluating high frequency signal components [4].

The functional significance or resistance of a coronary stenosis can be described more accurately by simultaneous measurement of intracoronary blood flow and the pressure gradient caused by the obstruction [2]. Meas- urements of flow-pressure relationships have been performed in animals $[5,6]$ and in patients $[7,8]$. However, simultaneous measurements are not used in clinical routine so far, because two sensor wires are needed and the evaluation of the combined measurements is not standardized yet and requires manual editing.

The aim of this work is twofold: Firstly, the initial verification of an evaluation procedure for combined pressure and flow measurements that is feasible for clinical practice. Secondly, the development of a robust pressure based parameter which is independent of maximum hyperemia and can complement the information obtained with FFR in those cases where flow measurements are not feasible. This is done by spectral correlation of pressure signals acquired proximal and distal of the lesions.

\section{Methods}

\subsection{Clinical setup}

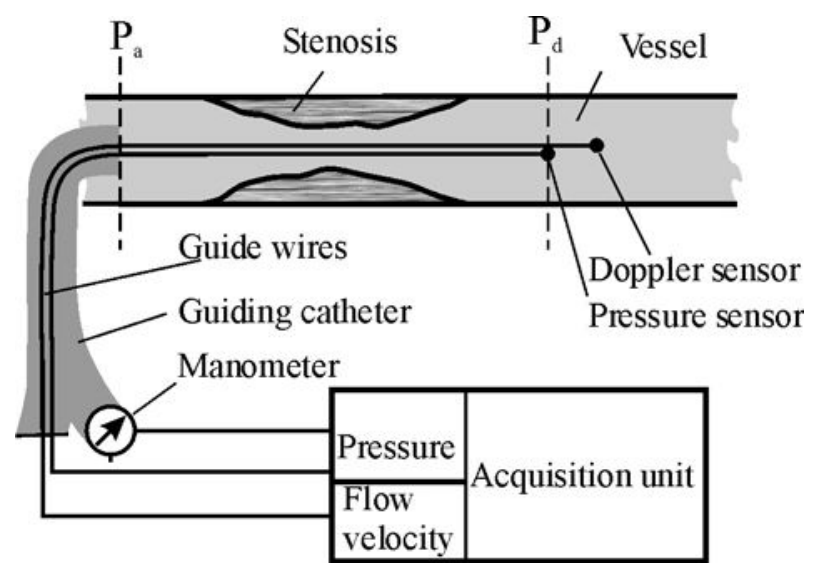

Figure 1: Measurement setup for intracoronary pressure and flow velocity signals.

The measurements were performed with informed patient's consent during routine procedures in the catheter lab. Patients who were scheduled for diagnostic catheterization or interventional procedures were selected for this trial. The aortic pressure $p_{\text {a }}$ proximal of a stenosis was measured using a fluid filled guiding catheter.

A pressure guide wire (Radi pressure wire, Radi, Sweden 
or Wavewire, Jomed, USA) was used for measuring the pressure $p_{\mathrm{d}}$ distal of a lesion. Prior to the examinations the sensor of the pressure wire was placed near the outlet of the guiding catheter and the two pressure signals were equalized to avoid a signal offset. For flow velocity measurements a Doppler guide wire (Flowire, Jomed, USA) was also placed distally of the lesion in the vicinity of the pressure sensor. Figure 1 shows a schematic of the measurement setup. Maximum flow was induced by continuous intravenous infusion of $140 \mu \mathrm{g}$ adenosine $/ \mathrm{kg} / \mathrm{min}$. Thus, a significant pressure gradient $\Delta p=p_{a}-p_{d}$ occurs in the presence of a stenosis.

Over a period of two minutes the two pressure signals and the flow velocity were recorded simultaneously, along with an electrocardiogram (ecg). A typical set of signals is shown in Figure 2.

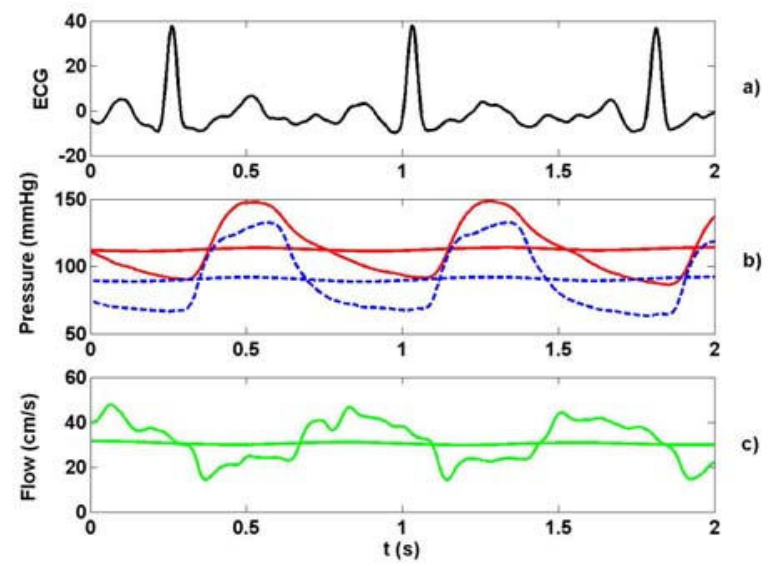

Figure 2: Typical dataset consisting of (a) ecg signal, (b) proximal (solid line) and distal (dashed line) pressure signals and (c) flow velocity, shown for two heart cycles. Mean values of pressure and flow are displayed as straight lines.

\subsection{Data processing and analysis}

The data was digitized at $200 \mathrm{~Hz}$ and stored on a personal computer for offline processing. Data analysis was performed with the Matlab ${ }^{\circledR}$ software package (The Math Works Inc., USA).

Prior to the analysis the data was low pass filtered to remove unwanted noise. A moving average filter was used to compute average pressure and flow signals. For each patient a time window was selected visually for data analysis. In this time window the intracoronary flow changed from resting conditions to intermediate flow and maximum hyperemia. In [2,7] it was shown that the flowpressure gradient relationship of a stenosis can be approximated by a $2^{\text {nd }}$ order polynomial equation derived from fluid dynamics in rigid tubes:

$\Delta p=a \cdot V+b \cdot V^{2}$
Here, $\Delta p$ denotes the pressure gradient and $V$ denotes the flow velocity. The values $a$ and $b$ are constants that have to be determined for stenosis characterization. However, this evaluation has to be performed in certain parts of the heart cycle [2], which requires manual selection of signal windows for each cycle. This approach is time consuming and might not be feasible in clinical practice. Therefore, in this work the average pressure gradient $\Delta \bar{p}$ was plotted against average flow velocity $\bar{V}$, which has been previously investigated in animal models [6]. This approach does not require manual editing. Linear regression was applied to the data and the graph of the resulting straight line was determined using the equation:

$$
\Delta \bar{p}=m \cdot \bar{V}+n
$$

The value $m$ denotes the slope of the straight line, which was used as a parameter to characterize the resistance $R_{s}$ of a stenosis. The value $n$ denotes the ordinate intercept (zero flow), which was not considered in the subsequent analysis.

For the derivation of the proposed new spectral parameter, signal windows at resting conditions and at maximum hyperemia were evaluated separately, in order to test the hypothesis that hyperemia is not a prerequisite for this method. After removing the mean values, the instantaneous pressure signals were Fourier transformed. The frequency range of the signals was limited from $0 \mathrm{~Hz}$ to the $8^{\text {th }}$ harmonic of the fundamental frequency. The cross correlation function (CCF) of the spectra was used to assess the stenosis severity. The normalized CCF can be expressed as:

$$
R(k)=\frac{\sum_{i=1}^{N} \mathrm{P}_{d}(i) \cdot P_{a}^{*}(i+k)}{\sqrt{\sum_{i=1}^{N}\left|P_{d}(i)\right|^{2} \cdot \sum_{i=1}^{N}\left|P_{a}(i)\right|^{2}}}
$$

In this equation, $P_{\mathrm{d}}$ and $P_{\mathrm{a}}$ denote the discrete Fourier transforms of the pressure signals, the window size is $N$ samples, which was chosen to $N=1000$ in the subsequent analysis. The maximum absolute value of the $\mathrm{CCF}$ $\max \{|R(k)|\}$ was used as a parameter for the degree of obstruction.

For verification purposes the results were compared to FFR, which is currently the most accurate clinically established method for functional stenosis assessment. It is defined as $F F R=\bar{p}_{d} / \bar{p}_{a}$, where $\bar{p}_{d}$ and $\bar{p}_{a}$ are the mean pressure values proximal and distal of a stenosis, measured at maximum hyperemia. Stenoses with $F F R<0.75$ are functionally significant [9] and usually treated by percutaneous intraluminal angioplasty (PTCA). 


\section{Results}

The severity of the examined stenoses ranged from angiographically mild to severe obstructions, with FFR values between 0.96 and 0.66 . In all patients maximum hyperemia was induced by intravenous infusion of adenosine. In Figure 3 the mean pressure gradient is plotted versus the mean flow velocity. Four datasets are exemplarily shown. In each set the flow ranges from resting conditions to hyperemia, which is patient specific. Due to the presence of stenoses the pressure gradient also rises. The graphs reflect different degrees of hemodynamic significance. Stenosis resistance changes from moderate to high with increasing slope, which was verified by FFR and QCA. The correlation of the linear regression is $r>0.95$ for all graphs. Figure 4 shows the comparison of the estimated slopes $m$ with FFR for all ten patients. As expected, the slopes increase with stenosis severity, as indicated by FFR.

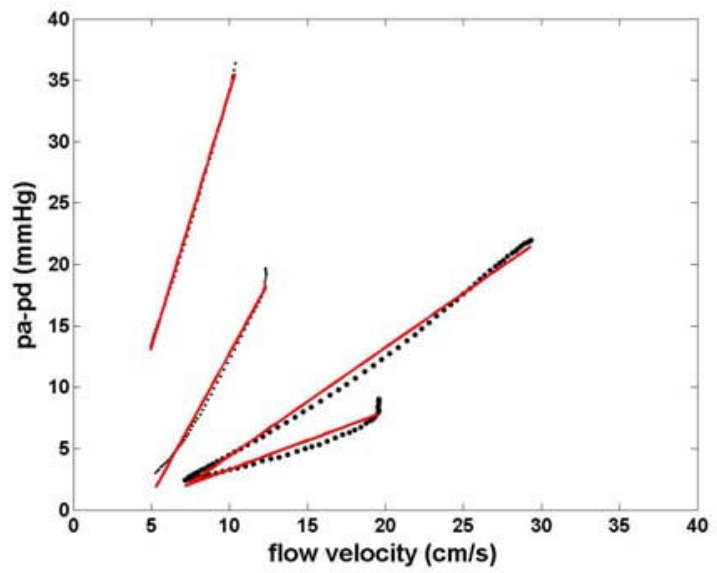

Figure 3: Flow-pressure gradient relationship for four patients with different degrees of obstruction. Linear regression (solid lines) is applied to the data (dotted lines)

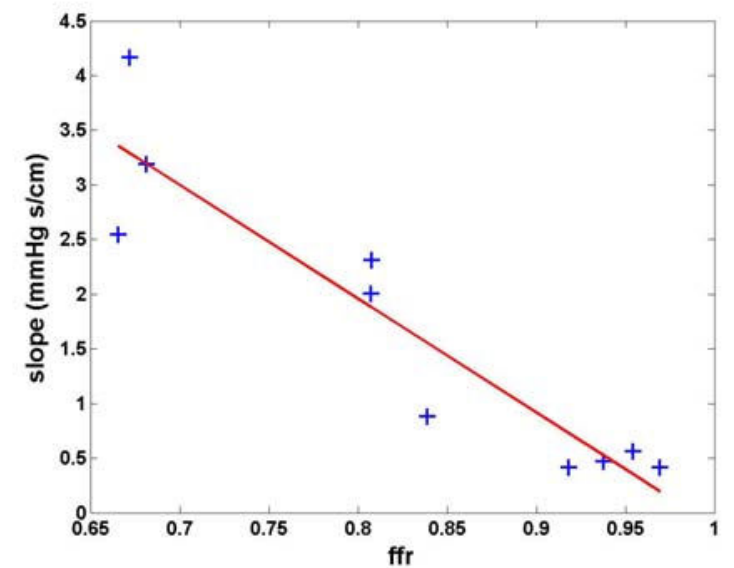

Figure 4: Estimated slopes $m=\Delta \bar{P} / \bar{V}$ plotted versus FFR $(r=0.93)$
The results for the spectral cross correlation are shown in Figures 5and 6. Here, the maximum of the $\mathrm{CCF}$ is plotted versus FFR for resting conditions (Figure 5) and the state of maximum flow (Figure 6). The linear regressions show a correlation of $r=0.89$ and $r=0.91$, respectively. Note that FFR was measured at maximum flow conditions in both cases.

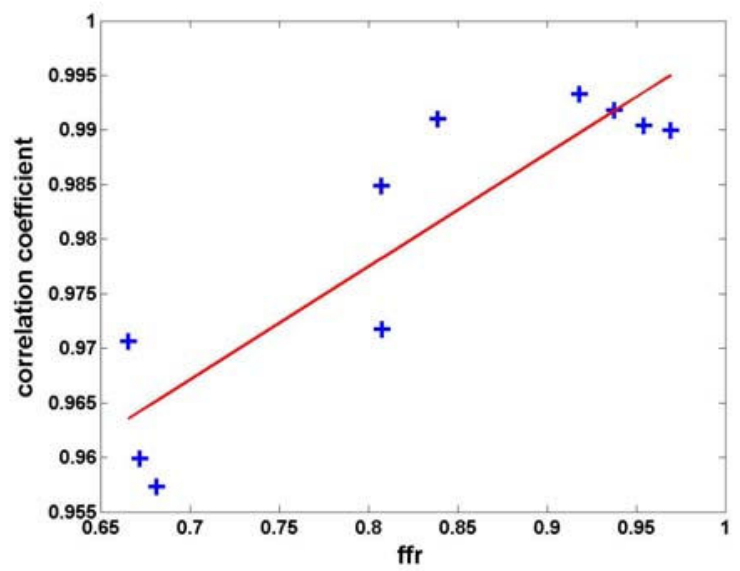

Figure 5: Maximum of the normalized cross correlation function at resting flow conditions $\left(\mathrm{CCF}_{\text {rest }}\right)$ plotted versus FFR $(r=0.89)$

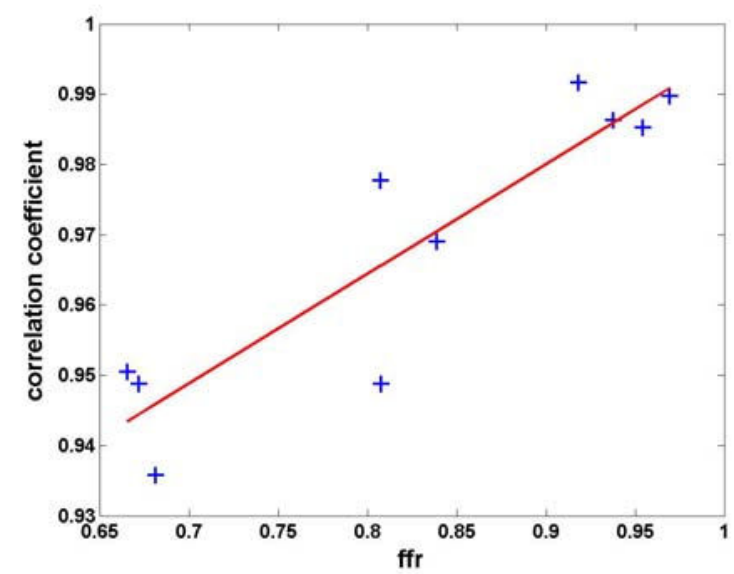

Figure 6: Maximum of the normalized cross correlation function at maximum hyperemia $\left(\mathrm{CCF}_{\text {hyp })}\right.$ plotted versus FFR $(r=0.91)$

\section{Discussion}

The results show a high correlation between FFR and the slope of the mean flow-pressure gradient relationship. As expected the slopes increase with stenosis severity. This suggests that the flow-pressure relationship derived from mean values is a useful indicator to characterize functional stenoses severity. The evaluation of mean values is a robust method that does not require manual editing. Furthermore, the slope as a single parameter for le- 
sion assessment seems to be sufficient and more convenient as opposed to the analysis of $2^{\text {nd }}$ order polynomials.

It is known from fluid dynamics that the simultaneous measurement of pressure and flow velocity describes a lesion most accurately [2,5]. Thus, this method might have advantages in comparison to FFR in some cases. This fact cannot be shown in this study, however, because FFR is currently the best validated physiological index. In order to verify the advantages of combined pressure and flow measurements, additional clinical trials with larger patient populations are needed.

The maxima of the spectral cross correlation functions decrease with lower FFR values as shown in Figures 5 and 6. The evaluation at maximum flow shows a higher correlation with the regression curve than the results at resting conditions. However, the correlation $(r=0.89)$ of the linear regression for resting conditions suggests that spectral cross correlation can provide a useful parameter also for the nonhyperemic state. Thus, it can be used in cases where maximum flow cannot be induced. The spectral evaluation of pressure signals was previously investigated by Brosh et. al. [4]. This approach evaluates high frequency components in the region of the dichrotic notch [4], which is characteristic for arterial pressure waveforms. However, sometimes intracoronary pressure measurements do not show a prominent dichrotic notch. In those cases the cross correlation of signal spectra might be more accurate because this method does not require the selection of a region within a pressure cycle.

\section{Conclusions}

This work presented first clinical results of intracoronary pressure and blood flow velocity analysis for the assessment of coronary stenosis severity. The slope of the flow-pressure relationship calculated from mean values was used as a parameter for stenosis characterization. This evaluation method proved to be robust and did not require manual editing. The comparison with the fractional flow reserve showed a high correlation, which suggests that this method is feasible in clinical practice.

Furthermore, spectral analysis of pressure waveforms was investigated. The maximum of the spectral cross correlation function showed a correlation to FFR of $r \geq 0.89$ both at resting conditions and maximum hyperemia. Therefore, this parameter can potentially be used in cases where induction of hyperemia is not possible. This has to be verified in subsequent clinical studies with a higher patient population.

Future work will be dedicated to the quantification of the parameters derived in this work. In comparison with the best validated approach in the evaluation of coronary artery stenosis severity (FFR), upcoming clinical trials will have to determine suitable threshold values as guidelines for clinical decisions for subsequent interventional procedures.

\section{Acknowledgements}

A project of the Ruhr Center of Competence for Medical Engineering (KMR), funded by the German Federal Ministry of Education and Research $(b m b+f$ grant No. 13N8079)

\section{References}

[1] Pijls NH, De Bruyne B, Peels K, Van Der Voort PH, Bonnier HJ, Bartunek JKJ, Koolen JJ. Measurement of fractional flow reserve to assess the functional severity of coronary-artery stenoses. New England Journal of Medicine 1996;334:1703-8.

[2] Gould KL. Coronary artery stenosis and reversing atherosclerosis. 2 ed. Arnold, 1999.

[3] Pijls NH, De Bruyne B, Bech GJ, Liistro F, Heyndrickx GR, Bonnier HJ, KoolenJJ. Coronary pressure measurement to assess the hemodynamic significance of serial stenoses within one coronary artery: validation in humans. Circulation 2000;102:2371-7.

[4] Brosh D, Higano ST, Slepian MJ, Miller HI, Kern MJ, Lennon RJ, Holmes DR, Lerman A. Pulse transmission coefficient: a novel nonhyperemic parameter for assessing the physiological significance of coronary artery stenoses. Journal of the American College of Cardiology 2002;39:1012-9.

[5] Gould KL. Pressure-flow characteristics of coronary stenoses in unsedated dogs at rest and during coronary vasodilation. Circulation Research 1978;43:242-53.

[6] Gould KL, Lipscomb K, Calvert C. Compensatory changes of the distal coronary vascular bed during progressive coronary constriction. Circulation 1975;51:1085-94.

[7] Marques KM, Spruijt HJ, Boer C, Westerhof N, Visser CA, Visser FC. The diastolic flow-pressure gradient relation in coronary stenoses in humans. Journal of the American College of Cardiology 2002;39:1630-6.

[8] Bojara W, Perrey C, Lindstaedt M, Fadgyas T, Lemke B, Ermert H. Charakterisierung des funktionellen Schweregrades von Koronarstenosen anhand simultaner intrakoronarer Druck- und Flussgeschwindigkeitsmessungen. Biomedizinische Technik 2001;46:352-53.

[9] Bech GJ, Droste H, Pijls NH, De Bruyne B, Bonnier JJ, Michels HR, Peels KH, Koolen JJ. Value of fractional flow reserve in making decisions about bypass surgery for equivocal left main coronary artery disease. Heart 2001;86:547-52.

Address for correspondence:

Christian Perrey

Institute of High Frequency Engineering

Ruhr University Bochum, IC 6/133

44780 Bochum, Germany

Christian.Perrey@rub.de 